\title{
Molecular Imaging: Radiopharmaceuticals for PET and SPECT
}

Shankar Vallabhajosula

Berlin, Germany: Springer-Verlag, 2009, 371 pages, \$179

Molecular Imaging: Radiopharmaceuticals for PET and SPECT is a well-written compendium of chapters that cover a wide range of topics regarding the use of radiopharmaceuticals in SPECT and PET. Dr. Vallabhajosula has effectively summarized the basics of chemistry, radiochemistry, radiation dosimetry, cell and molecular biology, and the physics of imaging and has outlined the regulatory processes required for drug development, including an exploratory Investigational New Drug (e-IND), good manufacturing processes, and the applicable chapters of the U.S. Pharmacopeia. Without any fellow editors or contributors, the author presents and discusses all aspects of radionuclide imaging, including the chemistry and applications for current and cutting-edge SPECT and PET radiopharmaceuticals in a single-volume, 371-page, dynamically illustrated textbook. It is intended for a broad audience that would include practicing physicians, residents, fellows, nuclear pharmacists, and nuclear medicine technologists.

This contemporary, hardcover book contains 20 chapters. Chapters 1 through 9 review the basic sciences. Chapters 10 through 14 include radiopharmaceutical chemistry, quality control of radiopharmaceuticals, and pharmacokinetics. The application of radiopharmaceuticals in molecular imaging for oncology, neurology, cardiology, and gene expression is contained in chapters 15 through 18. Chapters 19 and 20 are reserved for discussions of radiation dosimetry and drug development, including regulatory issues, such as the Investigational New Drug process.

Two specific aspects of the book give the text character and hold the reader's interest. The first is the quotations by prominent scientists at the beginning of each chapter. The quotations offered by individuals such as Einstein, Curie, and Crick range from humorous to thought-provoking. The second feature of interest is the historic perspective added by the author. The first 6 chapters contain some level of historic reference that is not essential for understanding the content of the chapter but adds depth. The author admits in the preface that the second chapter, which provides a brief history of the atom, is not essential; however, the historic

COPYRIGHT (c) 2010 by the Society of Nuclear Medicine, Inc. DOI: 10.2967/jnumed.109.072645 interludes provide an interest level for the reader that is not usually found in texts of this genre.

Because the book offers a concise review of basic science, it makes an excellent tool for the student of molecular imaging and also offers a reference for the experienced practitioner who might feel a bit rusty in organic chemistry or molecular biology. For instance, chapter 4-Radioactivitycontains a review of the types of radioactive decay and radioactive decay equations. Chapter 5 is a review of radionuclide production, including reactors, cyclotrons, and generator systems. Chapter 6 is a review of PET and SPECT scanners that includes radiation detectors, Anger cameras, PET scanners, and small-animal imaging systems. The principles of chemistry found in chapter 7 range from the basics of the periodic table through organic and biochemistry. Cell and molecular biology are presented in chapter 8 , and the basics of radiopharmaceuticals are offered in chapter 9.

With a foundation of the basic sciences established, chapter 10 begins with the chemistry of radiohalogens to acquaint the student of molecular imaging with information on fluorination and iodination. Because fluorination is such an essential part of PET today, a large portion of the chapter is devoted to labeling with ${ }^{18} \mathrm{~F}$, including nucleophilic and electrophilic reactions. Chapter 11 discusses the chemistry of carbon, nitrogen, and oxygen as they relate to PET. The chemistry of metal radionuclides, metal chelation, and technetium as it relates to SPECT and PET is discussed in chapter 12. Quality assurance, current good manufacturing practices, and quality control are discussed in chapter 13 . Chapter 14 is a discussion on pharmacokinetics and modeling, including quantitation, binding, and kinetics.

Clinical applications follow the radiopharmaceutical chemistry section. The greatest depth of information on clinical applications is provided in chapter 16, Neurology and Psychiatry. A wealth of information is presented on neuroscience and the use of molecular imaging radiopharmaceuticals for functional brain imaging, neurology, and psychiatric disorders. Chapter 15 discusses tumor pathology, biology, genetic changes, and imaging. The basis of cardiology imaging is presented in chapter 17, and imaging 
in relation to gene expression and cell trafficking is discussed in chapter 18.

For those interested in the initial pursuit of the development of a molecular imaging agent or who simply desire a better understanding of this process, chapter 19 presents the basic concepts of radiation dosimetry (including radiation protection). Chapter 20 continues with an outline of regulatory issues associated with drug development, including the Food and Drug Administration, the Radioactive Drug Research Committee, the Investigational New Drug application, including the new e-IND, and the U.S. Pharmacopeia.

Overall, this textbook would make a valuable and interesting addition to the library of anyone who is studying or has an interest in the current SPECT and PET radiopharmaceuticals used in molecular imaging.
Dr. Vallabhajosula has a vast experience in this field and is eminently qualified to author Molecular Imaging: Radiopharmaceuticals for PET and SPECT. There is a growing need for such a reference text as investigators from multiple scientific fields come together to work in this field. Dr. Vallabhajosula deserves our congratulations and gratitude for using his experience over 3 decades to bring this text to publication.

\section{Jeff Clanton}

Martin P. Sandler*

${ }^{*}$ Vanderbilt University Medical Center

CCC-1108 MCN

21st St. and Garland Ave.

Nashville, TN 37232

E-mail: martin.sandler@vanderbilt.edu 